\title{
Enterococcus faecium WB2000 Inhibits Biofilm Formation by Oral Cariogenic Streptococci
}

\author{
Nao Suzuki, Masahiro Yoneda, Yuko Hatano, Tomoyuki Iwamoto, \\ Yosuke Masuo, and Takao Hirofuji \\ Section of General Dentistry, Department of General Dentistry, Fukuoka Dental College, 2-15-1 Tamura, \\ Sawara-ku, Fukuoka 801-0193, Japan \\ Correspondence should be addressed to Nao Suzuki, naojsz@college.fdcnet.ac.jp
}

Received 2 March 2011; Revised 15 June 2011; Accepted 12 August 2011

Academic Editor: Marilia A. R. Buzalaf

Copyright (๑) 2011 Nao Suzuki et al. This is an open access article distributed under the Creative Commons Attribution License, which permits unrestricted use, distribution, and reproduction in any medium, provided the original work is properly cited.

This study investigated the inhibitory effect of probiotic Enterococcus faecium WB2000 on biofilm formation by cariogenic streptococci. The ability of E. faecium WB2000 and JCM5804 and Enterococcus faecalis JCM5803 to inhibit biofilm formation by seven laboratory oral streptococcal strains and 13 clinical mutans streptococcal strains was assayed. The Enterococcal strains inhibited biofilm formation in dual cultures with the mutans streptococcal strains Streptococcus mutans Xc and Streptococcus sobrinus JCM5176 $(P<0.05)$, but not with the noncariogenic streptococcal strains. Enterococcus faecium WB2000 inhibited biofilm formation by $90.0 \%(9 / 10)$ of the clinical S. mutans strains and $100 \%(3 / 3)$ of the clinical S. sobrinus strains. After culturing, the $\mathrm{pH}$ did not differ between single and dual cultures. The viable counts of floating mutans streptococci were lower in dual cultures with E. faecium WB2000 than in single cultures. Enterococcus faecium WB2000 acted as a probiotic bacterial inhibitor of cariogenic streptococcal biofilm formation.

\section{Introduction}

Dental caries is a very common chronic disease arising from the interplay among the oral flora, teeth, and dietary factors. The major etiological players are the two $\alpha$-hemolytic "mutans group" streptococci: Streptococcus mutans and Streptococcus sobrinus $[1,2]$. These bacteria adhere tenaciously to glucan-coated surfaces, produce large amounts of extracellular polysaccharides, and are highly acidogenic and acid tolerant $[3,4]$. The colonization of dental plaque by S. mutans and S. sobrinus plays a causative role in dental caries. Sugar metabolism is central to the behavior of mutans streptococci, and sucrose, the most cariogenic dietary carbohydrate, is used to produce the extracellular polysaccharides that form the biofilm matrix, which aids in the association of mutans streptococci with dental plaque. Once the mutans streptococci biofilm becomes part of the dental plaque, the acidic byproducts of sugar fermentation dissolve tooth enamel, eventually resulting in dental caries [4]. Dental plaque contains numerous genetically distinct types of bacteria that live in close juxtaposition on host surfaces [5]. The "viridans group" streptococci, such as Streptococcus sanguinis, Streptococcus oralis, Streptococcus mitis, and Streptococcus gordonii, are noncariogenic streptococci. Together with Actinomyces spp., they play an important role as initial colonizers of the tooth surface in the formation of dental plaque [6].

Probiotic bacteria, defined as live microorganisms that confer a health benefit on the host when administered in adequate amounts (FAO/WHO 2001), are thought to play a role in the maintenance of oral health [7]. Studies examining the effects of oral probiotics demonstrated that the consumption of products containing Lactobacillus rhamnosus or Lactobacillus reuteri reduces the number of mutans streptococci in the oral cavity $[8,9]$. The use of probiotic tablets containing Bifidobacterium spp. normalized the microbiota in patients with periodontitis and gingivitis, as compared with a control group [10]. Lactobacillus salivarius taken orally improved the periodontal health of healthy volunteers, particularly smokers, and reduced the numbers of periodontal bacteria in subgingival plaque to patients with oral malodor reduced the malodor and bleeding on probing from the periodontal pocket [13]. Therefore, the probiotic bacteria 
used in the human oral cavity are mainly Lactobacillus spp. and Bifidobacterium spp., whereas Enterococcus strains have been used in human systemic health, especially as probiotic supplements to counter gastrointestinal diseases [14, 15], and they are widely used as veterinary feed supplements $[16,17]$.

Enterococci are facultative anaerobic, Gram-positive cocci that form a part of the normal gastrointestinal tract flora in animals and humans. They are also frequently found in fermented food that is consumed raw, such as cheese and meat, as well as in vegetables and olives [18]. The genus Enterococcus includes at least 23 species, two of which, E. faecalis and E. faecium, account for more than $95 \%$ of the clinically important isolates. Traditionally, they are regarded as low-grade pathogens, but they have emerged as an increasingly important cause of nosocomial infections [19]. They are rarely found in the healthy human oral cavity, yet $E$. faecalis appears to occur frequently, albeit in low numbers, in primary root canal infections, especially in teeth with coronal leakage [20]. The clinical use of E. faecalis and E. faecium during food fermentation and as probiotics demands careful safety evaluation [21]. Recently, Kumada et al. reported that E. faecium 129 BIO 3B (Biofermin Pharmaceutical, Kobe, Japan) inhibited biofilm formation in dual cultures with $S$. mutans, S. sobrinus, and S. sanguinis in vitro [22]. To examine the suitability of using probiotic E. faecium WB2000, which was isolated from the feces of a healthy human and is contained in the Japanese gastrointestinal agent Strong Wakamoto (Wakamoto Pharmaceutical, Tokyo, Japan), to maintain oral health, this study examined the inhibition of biofilm formation by viridans group streptococci or cariogenic mutans streptococci in dual cultures with $E$. faecium WB2000 using 96-well microtiter plate assays.

\section{Methods and Materials}

2.1. Bacterial Strains and Culture Conditions. Enterococcus faecium WB2000 previously classified as Streptococcus faecalis [23], which was provided by Wakamoto Pharmaceutical, as well as E. faecium JCM5804, E. faecalis JCM5803, S. mutans JCM5705 and Xc, S. sobrinus JCM5176, S. gordonii DL1 (Challis), S. sanguinis American Type Culture Collection (ATCC) 10556, S. oralis ATCC 10557, S. mitis ATCC 903, 10 clinical strains of $S$. mutans (SMW01, SMW03, SMW08, SMW09, SMW10, SMW11, SMW13, SMW15, SMW22, and SMF01), and three clinical strains of S. sobrinus (SSW07, SSW14, and SSW24) were examined. The 13 clinical isolates of mutans streptococci, which have the ability to form, biofilms, were isolated from the saliva of 35 healthy adult volunteers (23 males and 12 females, aged 25-61 years, with a mean age of $38.5 \pm 9.8$ years $[ \pm$ SD] $)$ using selective medium (CRT bacteria; Ivoclar Vivadent AG, Schaan, Principality of Liechtenstein) and the polymerase chain reaction. The volunteers consisted of workers employed by Wakamoto Pharmaceutical Co. (Tokyo, Japan) and coauthors at the Fukuoka Dental College. Permission for this study was obtained from the Ethics Committee for Clinical Research at Wakamoto Pharmaceutical Co. and at the Fukuoka Dental College (approval number, 2010-02 and 163, resp.). After growth to stationary phase, the bacteria were suspended into skim milk and stored as $1-\mathrm{mL}$ aliquots in sterile tubes at $-80^{\circ} \mathrm{C}$.

The ability of the clinical mutans streptococcal isolates to form a biofilm in a 24-well (flat-bottom) microtiter plate (Sumitomo Bakelite Co., Tokyo, Japan) was determined as follows. A single colony was cultured in $1 \mathrm{~mL}$ of GAM both with $2.0 \%$ sucrose for $24 \mathrm{~h}$, and the supernatant was discarded. The well was rinsed three times with sterile distilled water (d-water), air-dried, and stained with $0.1 \%$ crystal violet solution for $15 \mathrm{~min}$. After staining, the well was rinsed with d-water to remove excess dye and air-dried. The presence of a biofilm mass was confirmed by visual assessment.

2.2. Human Saliva Preparation. Whole saliva samples were collected from three healthy human participants (mean age, $31.7 \pm 5.5$ years) by their chewing paraffin gum. The mixed saliva was centrifuged and $10000 \times \mathrm{g}$ for $20 \mathrm{~min}$ at $4^{\circ} \mathrm{C}$, and the supernatant was incubated at $56^{\circ} \mathrm{C}$ for $30 \mathrm{~min}$ to inactivate degradative enzymes. The samples were sterilized using a sterile membrane filter (pore size, $0.22 \mu \mathrm{m}$; Millipore, Billerica, MA, USA) and used immediately for the biofilm formation assay.

2.3. Biofilm Formation Assay in 96-Well Microtiter Plates. Biofilm formation by each strain was assayed using a described method [22]. To start the biofilm formation assay, precultures of each bacterium stored at $-80^{\circ} \mathrm{C}$ were grown in $10 \mathrm{~mL}$ of brain heart infusion (BHI) medium (Difco Laboratories, Detroit, MI, USA) for $24 \mathrm{~h}$ at $37^{\circ} \mathrm{C}$ to full growth. To evaluate biofilm formation in cocultures of Enterococcus spp. and oral Streptococcus spp., $20 \mu \mathrm{L}$ of an enterococcal cell suspension and $20 \mu \mathrm{L}$ of the other bacterial cell suspension were mixed in a well of a 96-well (flat-bottom) microtiter plate (Nunc A/S, Roskilde, Denmark), along with $160 \mu \mathrm{L}$ of tryptic soy broth (without dextrose, supplemented with 0.25\% sucrose; TSBS; Wako Pure Chemical Industries, Osaka, Japan), after coating the plates with whole saliva for $30 \mathrm{~min}$ at $37^{\circ} \mathrm{C}$. To evaluate biofilm formation by single cultures, $20 \mu \mathrm{L}$ of bacterial cell suspension and $180 \mu \mathrm{L}$ of TSBS were added to each well. The plates were incubated at $37^{\circ} \mathrm{C}$ for $16 \mathrm{~h}$ under anaerobic conditions, and the liquid medium was removed. The wells were rinsed a second time with d-water, air-dried, and stained with $0.25 \%$ safranin for $15 \mathrm{~min}$. After staining, the plates were rinsed with dwater to remove excess dye and then air-dried. The biofilm mass was dissolved with ethanol, and the stained biofilm was quantified by measuring the absorbance at $492 \mathrm{~nm}$ using a microplate reader (Sunrise Rainbow Thermo; Tecan Group, Männedorf, Switzerland). The $\mathrm{pH}$ of the supernatant was determined before and after culturing for $16 \mathrm{~h}$.

2.4. Determination of the Viable Bacterial Count. To evaluate the change in bacterial cell numbers in the biofilm formation assay, diluted supernatant of the reaction mixture was cultured on GAM (Nissui, Tokyo, Japan) agar plates containing $2.0 \%$ sucrose for enterococci or on GAM agar plates containing $2.0 \%$ sucrose and 0.2 units $\mathrm{mL}^{-1}$ bacitracin (Sigma, St. Louis, MO, USA) for mutans group streptococci. 


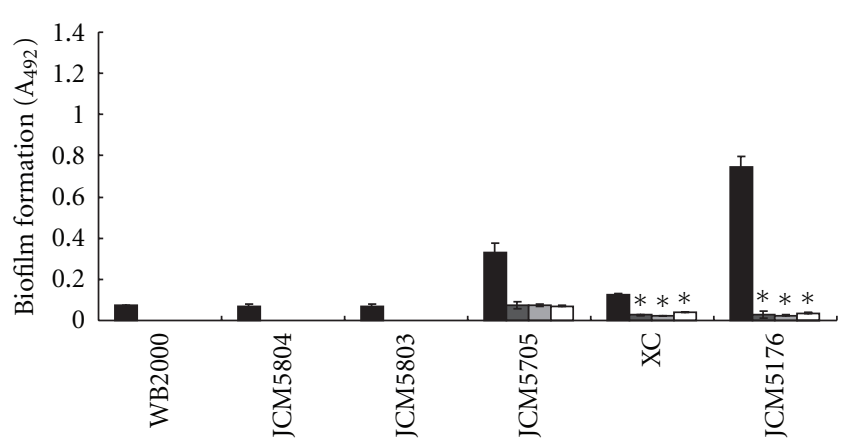

Figure 1: Inhibitory effects of E. faecium WB2000 and JCM5804 and E. faecalis JCM5803 on biofilm formation by laboratory mutans group streptococci. WB2000, E. faecium WB2000; JCM5804, E. faecium JCM5804; JCM5803, E. faecalis JCM5803; JCM5705, S. mutans JCM5705; Xc, S. mutans Xc; JCM5176, S. sobrinus JCM5176. Black bars: single culture; dark gray bars: dual culture with $E$. faecium WB2000; light gray bars: dual culture with E. faecium JCM5804; white bars: dual culture with $E$. faecalis JCM5803. ${ }^{*} P<0.05$ versus single culture.

After a 24 to $48 \mathrm{~h}$ incubation at $37^{\circ} \mathrm{C}$ under anaerobic conditions, the viable bacteria were counted.

2.5. Reproducibility and Statistical Analysis. Each assay was performed in at least three wells per plate, and at least three independent replicates were performed. The differences in biofilm formation by the laboratory streptococcal strains between single cultures and dual cultures with the enterococcal strains were analyzed using the Kruskal-Wallis test. For paired comparisons of biofilm formation using clinical mutans streptococci and E. faecium WB2000, the MannWhitney test was used. Differences at the 0.05 level were considered statistically significant.

\section{Results}

3.1. Effect of E. faecium and E. faecalis on Streptococcal Biofilms. The biofilm formation analyses of single cultures showed that E. faecium WB2000 and JCM5804 and E. faecalis JCM5803 produced little biofilm (Figure 1, lines 1-3). The viridans group streptococci also made little or no biofilm, except for S. oralis ATCC 10557 (data not shown). The Enterococcus strains did not inhibit biofilm formation by $S$. oralis, and a mixture of the other viridans group strains and Enterococcus strains did not make a biofilm. S. mutans JCM5705 and Xc and S. sobrinus JCM5176 made biofilms in single cultures (Figure 1, lanes 4-6), and every Enterococcus strain inhibited this biofilm formation. In particular, the biofilm formation by S. mutans Xc and S. sobrinus JCM5176 was significantly inhibited $(P<0.05)$.

Subsequently, 10 clinical $S$. mutans strains and three clinical S. sobrinus strains were examined. The single cultures resulted in various amounts of biofilm formation (Figure 2, lanes 1-13). The biofilm formation was reduced significantly in dual cultures of nine S. mutans $(90.0 \%)$ and three $S$. sobrinus $(100 \%)$ clinical strains with E. faecium WB2000. E. faecium WB2000 and JCM5804 and E. faecalis JCM 5803 had

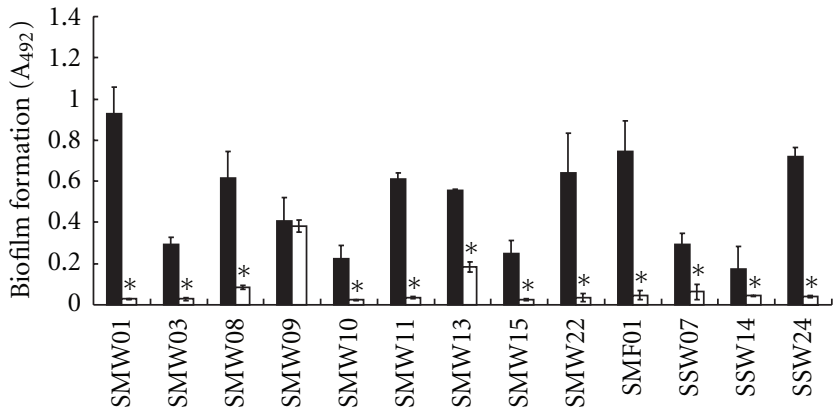

FIgURE 2: Inhibitory effects of E. faecium WB2000 on biofilm formation by clinical mutans group streptococci. SMWs and SMF, clinical S. mutans strains; SSWs, clinical S. sobrinus strains. Black bars: single culture; white bars: dual culture with E. faecium WB2000. ${ }^{*} P<0.05$ versus single culture.

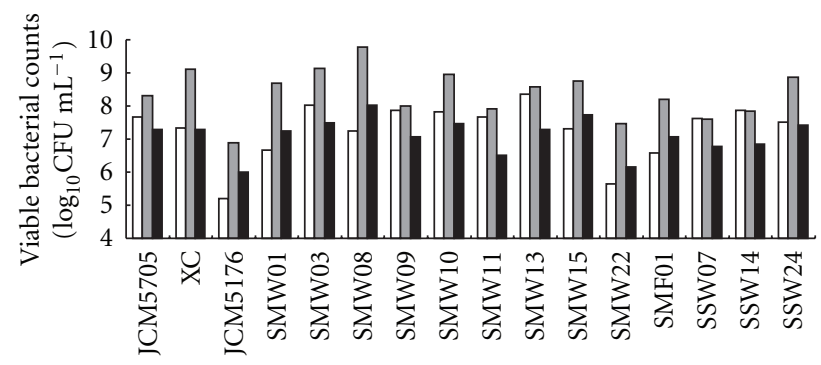

Figure 3: Viable bacterial counts of strains of mutans group streptococci in single cultures at 0 and $16 \mathrm{~h}$ and in dual cultures with E. faecium WB2000 at $16 \mathrm{~h}\left(\log _{10} \mathrm{CFU} \mathrm{mL}^{-1}\right)$. JCM5705, S. mutans JCM5705; Xc, S. mutans Xc; JCM5176, S. sobrinus JCM5176; SMWs and SMF, clinical S. mutans strains; SSWs, clinical S. sobrinus strains.

similar effects on reducing biofilm formation. The $\mathrm{pH}$ in the single cultures of $S$. mutans and $S$. sobrinus ranged from 4.6 to 4.8 , and the $\mathrm{pH}$ in the single culture of E. faecium WB2000 was 4.9. The $\mathrm{pH}$ of the dual cultures of mutans streptococci with E. faecium WB2000 was 4.8-4.9.

3.2. Viable Bacterial Count. A viable count was performed to reveal whether the decrease in streptococcal biofilm formation was caused by the death of bacterial cells or by another factor such as inhibition of cell adhesion. Figures 3 and 4 show the viable bacterial counts of mutans group streptococci and E. faecium WB2000 in single cultures at 0 and $16 \mathrm{~h}$ and in dual cultures at $16 \mathrm{~h}$. After culturing, the numbers of all of the bacterial strains in the dual cultures were lower than those in single cultures. The numbers of nine of the mutans streptococci in dual cultures with $E$. faecium WB2000 at $16 \mathrm{~h}$ were reduced in comparison with those at $0 \mathrm{~h}$. The pattern of the increase or decrease in the numbers of streptococci and enterococci varied. In dual cultures of S. mutans SMW09 and E. faecium WB2000, in which biofilm formation was not inhibited, the number of E. faecium WB2000 was markedly reduced (Figure 4, lane 9). 


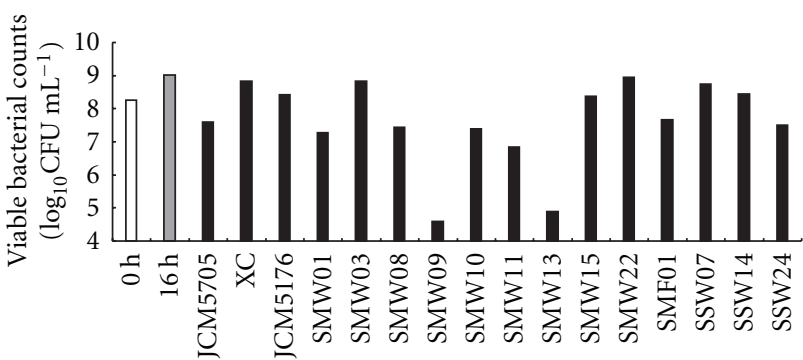

FIgURe 4: Viable bacterial counts of E. faecium WB2000 in single cultures at 0 and $16 \mathrm{~h}$ and in dual cultures with strains of mutans group streptococci at $16 \mathrm{~h}\left(\log _{10} \mathrm{CFU} \mathrm{mL} \mathrm{mL}^{-1}\right)$.

\section{Discussion}

This study evaluated the capacity of E. faecium WB2000 to inhibit biofilm formation by oral viridans group and mutans group streptococci. This organism markedly inhibited biofilm formation by mutans streptococci, as reported previously [22], but did not inhibit biofilm formation by S. oralis in the viridans group. The other three viridans group streptococci formed little or no biofilm in single cultures, and the combinations of viridans group streptococci with Enterococcus spp. resulted in equal or reduced total biofilm mass compared with the single enterococcal cultures. Standar et al. [24] reported that $S$. mitis failed to form biofilm structures in similar in vitro assays, but that the combination of $S$. mitis with $S$. mutans resulted in an increased total biofilm mass compared with cultures of $S$. mutans alone. Bacterial interactions are very important for biofilm formation. To confirm the specific inhibition of cariogenic streptococci by E. faecium WB2000, a future study should examine multiple bacteria.

In general, E. faecium WB2000 inhibited biofilm formation by the clinical mutans group streptococci, except for $S$. mutans SMW09, which was an exception to the probiotic effect of E. faecium WB2000. Nevertheless, planktonic numbers of mutans streptococcal strains cultured with E. faecium WB2000 were lower than those in single culture at $16 \mathrm{~h}$. These results indicate that E. faecium WB2000 in dual cultures possessed bacteriostatic or bactericidal activity against mutans streptococcal strains. The viable cell numbers of $E$. faecium WB2000 in dual cultures also varied. In particular, the number of E. faecium WB2000 cultured with S. mutans SMW09 decreased markedly by $16 \mathrm{~h}$. Overall, single cultures of the clinical strains produced various amounts of biofilm, and the increase or decrease in the number of bacteria in a reaction was strain dependent. These phenomena depended on differences in characteristics among the clinical strains and intercellular reactions. Deng et al. [25] examined the effects of S. mutans on E. faecalis biofilm formation using eight clinical E. faecalis strains with the ability to form biofilms and found differences among the strains.

A study of lactobacilli suggested that overgrowth and superinfection by a probiotic bacterium in the oral cavity are not a concern, although the stability of the exogenous bacteria in the oral cavity is unclear. In our previous study [13], the number of L. salivarius WB21 in saliva reached a peak on day 15 and did not increase further until day
29. Shimauchi et al. [11] reported that the populations of L. salivarius in saliva specimens from healthy volunteers in both test and placebo groups tended to decrease during the intervention period. Lactic acid bacteria commonly found in the oral cavity are Lactobacillus spp. and Bifidobacterium spp., whereas Enterococcus spp. are primarily in the large intestine and rarely in the oral cavity. The stability of $E$. faecium WB2000 and maintenance of its activity in a complex oral microflora should be examined in the future.

Enterococcus spp. are traditionally regarded as low-grade pathogens, but have been recovered from the oral cavity, mainly from the dental plaque of individuals with underlying diseases in whom opportunistic infections have occurred [26]. We examined the application of E. faecium WB2000 in oral health maintenance because this strain is harmless and has rarely been recovered from the oral cavity. For more than 50 years, E. faecium WB2000 has been used in traditional Japanese medicine (Strong Wakamoto ${ }^{\circledR}$ ) to treat gastrointestinal discomfort, and E. faecium has been used more often than E. faecalis to treat gastrointestinal trouble in humans. Compared with E. faecalis, E. faecium appears to pose a lower risk for use in foods because its strains generally possess fewer recognized virulence determinants [27] probably due to the presence of pheromone-responsive plasmids. A well-documented example of the safety of exogenous enterococci is E. faecium strain SF68, which has been used in pharmaceutical preparations to treat diarrhea $[15,28]$. This organism does not have any enterococcal virulence factors and is not able to adhere to vascular epithelial cells or endocardial cells [29]. No case of infection with probiotic E. faecium strains has ever been reported in humans. Although additional investigations are required to clarify the mechanism by which E. faecium inhibits biofilm formation by cariogenic mutans streptococci and to determine the stability of the E. faecium population the present study may provide valuable data regarding the use of an E. faecium strain as local therapy for oral infections.

\section{Acknowledgments}

This study was supported in part by a Grant-in-Aid for Young Scientists (no. 23792532 to Nao Suzuki), a Grant-inAid for Scientific Research (no. 23593078 to Takao Hirofuji), and by a Grant-in-Aid for Advanced Science Research from the Ministry of Education, Culture, Sports, Science and Technology of Japan. The authors thank Dr. H. Hirata and his team at Sagami Research Laboratories, Wakamoto Pharmaceutical Co. Ltd., for support and assistance during the study.

\section{References}

[1] J. M. Hardie and R. A. Whiley, "Plaque microbiology of crown caries," in Dental Plaque Revisited Cardiff, H. N. Newman and M. Wilson, Eds., pp. 283-204, Bioline, 1999.

[2] D. Beighton and S. R. Brailsfold, "Plaque microbiology of crown caries," in Dental Plaque Revisited Cardiff, H. N. Newman and M. Wilson, Eds., pp. 295-312, Bioline, 1999. 
[3] S. Hamada and H. D. Slade, "Biology, immunology, and cariogenicity of Streptococcus mutans," Microbiological Reviews, vol. 44, no. 2, pp. 331-384, 1980.

[4] W. J. Loesche, "Role of Streptococcus mutans in human dental decay," Microbiological Reviews, vol. 50, no. 4, pp. 353-380, 1986.

[5] P. E. Kolenbrander, "Oral microbial communities: biofilms, interactions, and genetic systems," Annual Review of Microbiology, vol. 54, pp. 413-437, 2000.

[6] R. J. Palmer Jr., S. M. Gordon, J. O. Cisar, and P. E. Kolenbrander, "Coaggregation-mediated interactions of streptococci and actinomyces detected in initial human dental plaque," Journal of Bacteriology, vol. 185, no. 11, pp. 3400-3409, 2003.

[7] J. H. Meurman and I. Stamatova, "Probiotics: contributions to oral health,” Oral Diseases, vol. 13, no. 5, pp. 443-451, 2007.

[8] A. J. Ahola, H. Yli-Knuuttila, T. Suomalainen et al., "Shortterm consumption of probiotic-containing cheese and its effect on dental caries risk factors," Archives of Oral Biology, vol. 47, no. 11, pp. 799-804, 2002.

[9] E. Çaglar, S. K. Cildir, S. Ergeneli, N. Sandalli, and S. Twetman, "Salivary mutans streptococci and lactobacilli levels after ingestion of the probiotic bacterium Lactobacillus reuteri ATCC 55730 by straws or tablets," Acta Odontologica Scandinavica, vol. 64, no. 5, pp. 314-318, 2006.

[10] A. I. Grudianov, N. A. Dmitrieva, and E. V. Fomenko, "Use of probiotics Bifidumbacterin and Acilact in tablets in therapy of periodontal inflammations," Stomatologiya, vol. 81, no. 1, pp. 39-43, 2002.

[11] H. Shimauchi, G. Mayanagi, S. Nakaya et al., "Improvement of periodontal condition by probiotics with Lactobacillus salivarius WB21: a randomized, double-blind, placebo-controlled study," Journal of Clinical Periodontology, vol. 35, no. 10, pp. 897-905, 2008.

[12] G. Mayanagi, M. Kimura, S. Nakaya et al., "Probiotic effects of orally administered Lactobacillus salivarius WB21containing tablets on periodontopathic bacteria: a doubleblinded, placebo-controlled, randomized clinical trial," Journal of Clinical Periodontology, vol. 36, no. 6, pp. 506-513, 2009.

[13] T. Iwamoto, N. Suzuki, K. Tanabe, T. Takeshita, and T. Hirofuji, "Effects of probiotic Lactobacillus salivarius WB21 on halitosis and oral health: an open-label pilot trial," Oral Surgery, Oral Medicine, Oral Pathology, Oral Radiology and Endodontology, vol. 110, no. 2, pp. 201-208, 2010.

[14] M. de Vrese and P. R. Marteau, "Probiotics and prebiotics: effects on diarrhea," Journal of Nutrition, vol. 137, no. 3, pp. 803S-811S, 2007.

[15] P. R. Marteau, M. de Vrese, C. J. Cellier, and J. Schrezenmeir, "Protection from gastrointestinal diseases with the use of probiotics," American Journal of Clinical Nutrition, vol. 73, no. 2, pp. 430S-436S, 2001.

[16] J. Benyacoub, G. L. Czarnecki-Maulden, C. Cavadini et al., "Supplementation of food with Enterococcus faecium (SF68) stimulates immune functions in young dogs," Journal of Nutrition, vol. 133, no. 4, pp. 1158-1162, 2003.

[17] L. Scharek, J. Guth, K. Reiter et al., "Influence of a probiotic Enterococcus faecium strain on development of the immune system of sows and piglets," Veterinary Immunology and Immunopathology, vol. 105, no. 1-2, pp. 151-161, 2005.

[18] P. Marteau, B. Messing, E. Arrigoni et al., "Do patients with short-bowel syndrome need a lactose-free diet?" Nutrition, vol. 13, no. 1, pp. 13-16, 1997.

[19] S. Sood, M. Malhotra, B. K. Das, and A. Kapil, "Enterococcal infections \& antimicrobial resistance," Indian Journal of Medical Research, vol. 128, no. 2, pp. 111-121, 2008.
[20] B. P. Gomes, E. T. Pinheiro, E. L. Sousa et al., "Enterococcus faecalis in dental root canals detected by culture and by polymerase chain reaction analysis," Oral Surgery, Oral Medicine, Oral Pathology, Oral Radiology and Endodontology, vol. 102, no. 2, pp. 247-253, 2006.

[21] T. J. Eaton and M. J. Gasson, "Molecular screening of Enterococcus virulence determinants and potential for genetic exchange between food and medical isolates," Applied and Environmental Microbiology, vol. 67, no. 4, pp. 1628-1635, 2001.

[22] M. Kumada, M. Motegi, R. Nakao et al., "Inhibiting effects of Enterococcus faecium non-biofilm strain on Streptococcus mutans biofilm formation," Journal of Microbiology, Immunology and Infection, vol. 42, no. 3, pp. 188-196, 2009.

[23] K. H. Schleifer and R. Kilpper-Balz, "Transfer of Streptococcus faecalis and Streptococcus faecium to the genus Enterococcus nom. rev. as Enterococcus faecalis comb. nov. and Enterococcus faecium comb. nov," International Journal of Systematic Bacteriology, vol. 34, no. 1, pp. 31-34, 1984.

[24] K. Standar, B. Kreikemeyer, S. Redanz, W. L. Münter, M. Laue, and A. Podbielski, "Setup of an in vitro test system for basic studies on biofilm behavior of mixed-species cultures with dental and periodontal pathogens," PLoS ONE, vol. 5, no. 10, Article ID e13135, 2010.

[25] D. M. Deng, M. A. Hoogenkamp, R. A. Exterkate et al., "Influence of Streptococcus mutans on Enterococcus faecalis biofilm formation," Journal of Endodontics, vol. 35, no. 9, pp. 1249-1252, 2009.

[26] J. Campbell, D. A. McGowan, and T. W. Macfarlane, "The prevalence of enterococci in the dental plaque of chronic hospital patients," British Journal of Oral Surgery, vol. 21, no. 3, pp. 171-174, 1983.

[27] C. M. Franz, M. E. Stiles, K. H. Schleifer, and W. H. Holzapfel, "Enterococci in foods-a conundrum for food safety," International Journal of Food Microbiology, vol. 88, no. 2-3, pp. 105-122, 2003.

[28] G. Reid, "The scientific basis for probiotic strains of Lactobacillus," Applied and Environmental Microbiology, vol. 65, no. 9, pp. 3763-3766, 1999.

[29] G. Chisari, A. M. lo Bue, L. Drago, R. Abbiati, and M. R. Gismondo, "Adhesion capacity of Entetococcus faecium (SF68) and Enterococcus faecalis to various substances," Giornale Italiano di Chemioterapia, vol. 39, no. 1-3, pp. 11-15, 1992. 


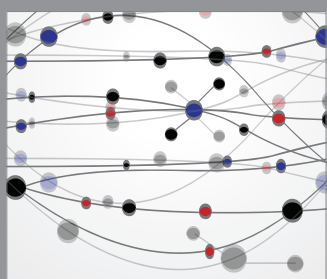

The Scientific World Journal
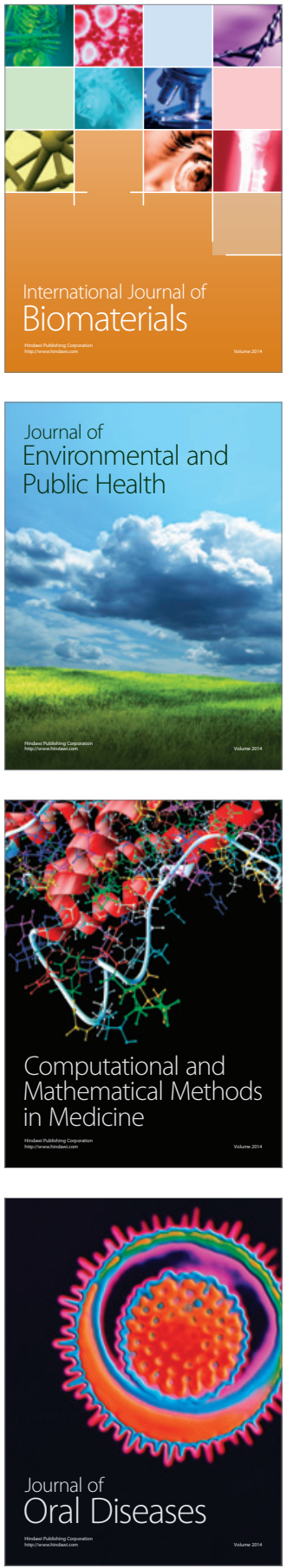
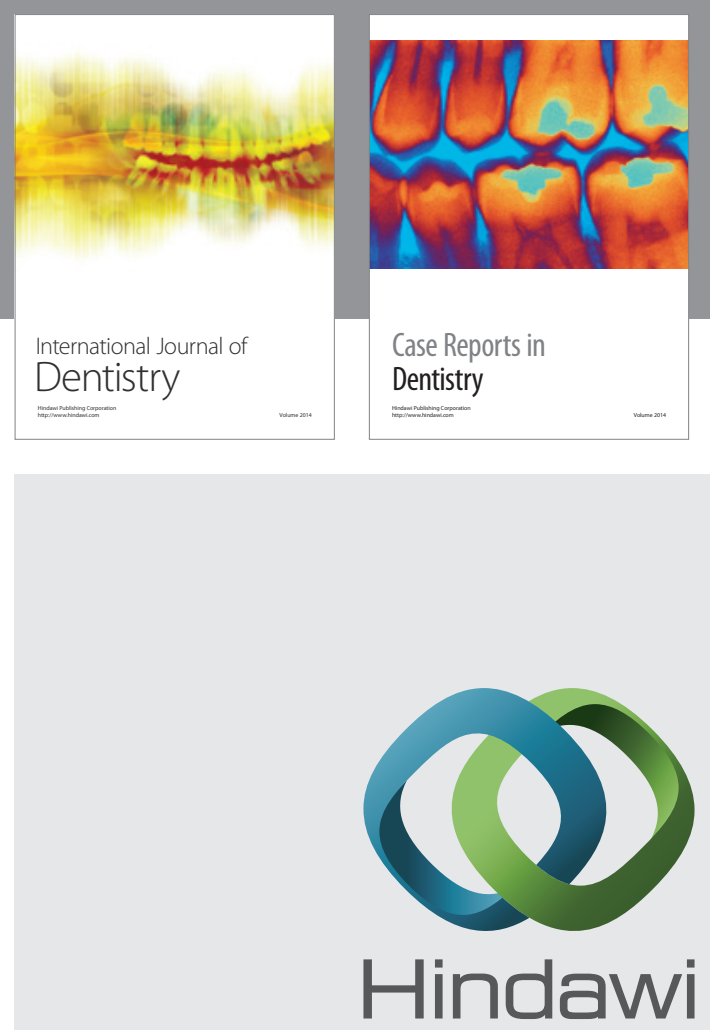

Submit your manuscripts at

http://www.hindawi.com
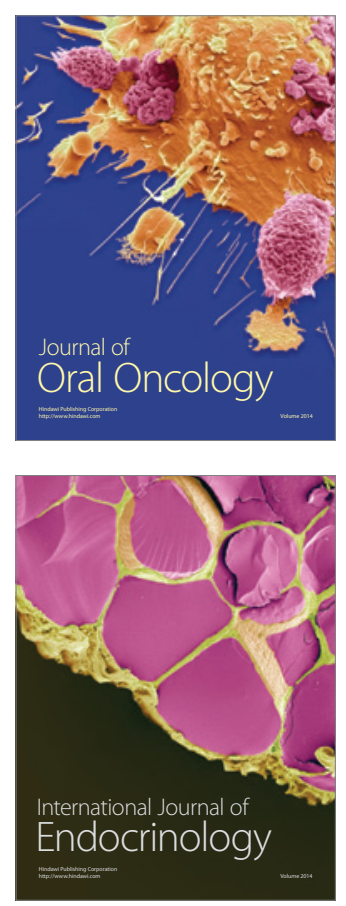
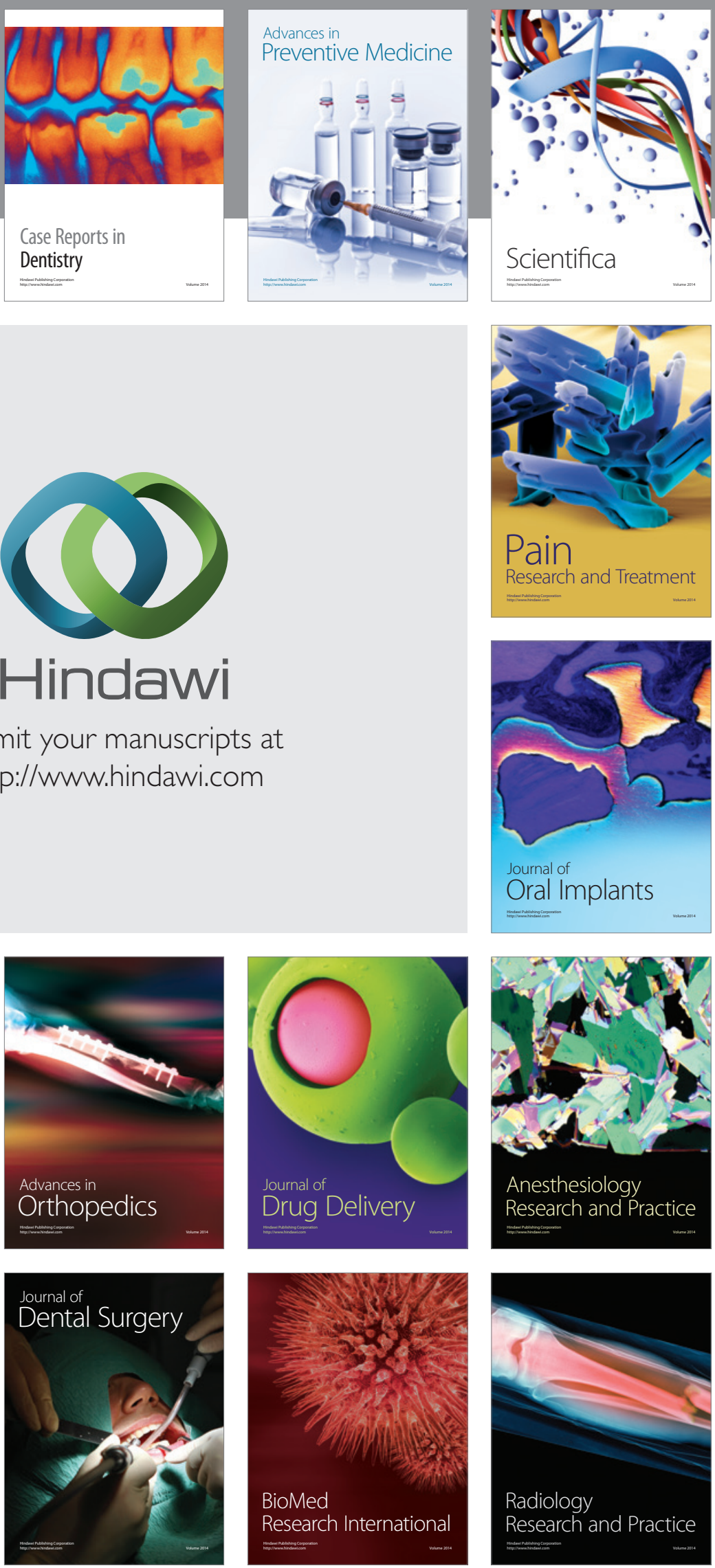